Cad.Est.Ling., Campinas, 51(1): 31-48, Jan./Jun. 2009

\title{
CONSTRUCCIONESDE INFINITIVOYSEMÁNTICAARGUMENTATIVA
}

\author{
MARÍAMARTAGARCÍANEGRONI \\ Universidad de Buenos Aires \\ SILVIARAMÍREZGELBES \\ Universidad de Buenos Aires, Universidad de San Andrés, IES en Lenguas Vivas
}

\begin{abstract}
RESUMO: Neste trabalho, analisamos distintas construções nominais de infinitivo em espanhol para mostrar a existência dos falsos infinitivos (Varela, 1979; Hernanz, 1999) que, como os dizeres e os seres, aceitam pluralização e entram em todas as construções nominais dos substantivos ordinários. Reconhecemos dois tipos: os falsos infinitivos de tipo $\mathrm{A}$, próximos às nominalizações dos verbos dos quais derivam, e os falsos infinitivos de tipo B, cujos sentidos parecem mais distantes do verbo de base.

No quadro da Teoria dos Blocos Semânticos, explicamos essa diferença e a aparente maior distância semântica que existe entre os falsos infinitivos tipo B e os verbos dos quais eles derivam. Finalmente e para poder fornecer uma explicação da escassa produtividade dos falsos infinitivos, propomos relacionálos com a aspectualidade: somente os verbos que contêm o traço homogeneidade/propriedade (como os estativos puros ou as atividades) podem desenvolver falsos infinitivos.

Concluímos que as construções de infinitivo representam um espaço interessante de pesquisa que demonstra, por um lado, a capacidade explicativa da TBS enquanto teoria semântica léxica e que, por outro lado, permite esclarecer os alcances das noções relativas à aspectualidade semântica.

Palavras chave: argumentação - nominalização - infinitivo - aspecto - teoria dos blocos semânticos

RESUMEN: En este trabajo, analizamos distintas construcciones nominales de infinitivo en español para mostrar la existencia de los falsos infinitivos (Varela, 1979; Hernanz, 1999) que, como los decires y los seres, aceptan la pluralización y entran en todas las construcciones nominales de los sustantivos ordinarios. Reconocemos dos tipos: los falsos infinitivos del tipo A, cercanos a las nominalizaciones de los verbos de los que derivan, y los falsos infinitivos del tipo B, cuyos sentidos parecen más alejados del verbo base. En el marco de la Teoría de los Bloques Semánticos, explicamos esa diferencia y damos cuenta de la aparente mayor distancia semántica que existe entre los falsos infinitivos tipo B y los verbos de los que ellos derivan. Finalmente y para poder brindar una explicación de la escasa productividad de los falsos infinitivos, proponemos relacionarlos con la aspectualidad: solo los verbos que contienen el rasgo homogeneidad/propiedad (como los estativos puros o las actividades) pueden desarrollar falsos infinitivos. En definitiva, las construcciones de infinitivo representan un espacio interesante de investigación que demuestra, por un lado, la capacidad explicativa de la TBS en tanto teoría semántica léxica y que, por el otro, permite arrojar luz sobre los alcances de las nociones relativas a la aspectualidad semántica.
\end{abstract}

Palabras clave:

\section{INTRODUCCIÓN}

Junto con el participio y el gerundio, el infinitivo constituye, como es sabido, una de las formas no flexivas del verbo: carece de los morfemas de tiempo, modo y aspecto, 
característicos de las formas conjugadas del verbo, y de los de número y persona, propios de la concordancia. Al igual que las otras dos formas no flexivas, el infinitivo se caracteriza por su doble pertenencia categorial: por un lado, se desempeña como verbo, pues acepta sus modificadores y adjuntos (sujeto, objeto directo e indirecto, circunstanciales, etc.) y, por el otro, como sustantivo, ya que admite los modificadores nominales (modificadores directos e indirectos, aposiciones, especificadores, etc.) ${ }^{1}$. De hecho, este carácter híbrido del infinitivo aparece subrayado por la cantidad de nombres que ha recibido. Así, por ejemplo y como bien recuerda Varela (1979: 531), los estoicos lo llamaban "verbo indeterminado"; Bello (1847), "derivado verbal sustantivo"; Lenz (1920), "verboide" y Gili y Gaya (1948), "sustantivo verbal" o "nombre del verbo".

Esta particular naturaleza que le permite, entonces, funcionar-sin perder su esencia verbal- como sustantivo, explica el hecho de que, ya desde los trabajos pioneros de Bello, se haya señalado la existencia de dos tipos de construcciones con infinitivo en español. Es el caso de (1) y (2):

1. (El) conocer Juan al ministro le abrió muchas puertas.

2. El masticar de Juan me molesta.

En ambos ejemplos, el infinitivo nuclea la construcción que funciona como sujeto de la oración (El conocer Juan al ministro / El masticar de Juan). Sin embargo, y tal como puede constatarse, en el primer caso, el infinitivo mantiene su funcionamiento verbal, puesto que soporta la coocurrencia de un sustantivo sujeto en nominativo (Juan) y de un objeto directo (al ministro) en acusativo. Asimismo, y tal como señala Varela (1979: 529), la especificación optativa $(e l)$-que señalamos por ello entre paréntesis- muestra que se trata de una estructura derivada de una oración plena, a saber:

\section{Juan conoce al ministro.}

Puede señalarse también que la construcción presente en (1) admite la voz pasiva (obsérvese en (4) el verbo en voz pasiva ((el) ser conocido) y la transformación del sujeto en complemento agente (por Juan)):

4. (El) ser conocido el ministro por Juan le abrió muchas puertas.

los tiempos compuestos (cf. en (5), haber conocido):

5. (El) haber conocido Juan al ministro le abrió muchas puertas.

así como también adjuntos circunstanciales (cf. en (6), el circunstancial de tiempo (hace unos años), o en (7), el de modo (profundamente)):

${ }^{1}$ En español, los infinitivos terminan en -ar, -er e -ir y pueden presentar una forma simple, que tiene sentido imperfectivo (por ejemplo, cantar, comer, vivir), y una forma compuesta, que tiene sentido perfectivo (por ejemplo, haber cantado, haber comido, haber vivido). 
6. (El) haber conocido Juan hace unos años al ministro le abrió muchas puertas.

7. (El) conocer profundamente al ministro le abrió muchas puertas.

A diferencia de (1), la construcción de (2) reviste un carácter más nominal: en efecto, en (2), la presencia del especificador es obligatoria (cf. imposibilidad de (8)) y, tal como se ve en (9) y en (10), el infinitivo puede, al igual que los sustantivos, coocurrir con cualquier determinante: ${ }^{2}$

8. *Masticar de Juan me molesta.

9. Su masticar me molesta.

10. Ese masticar con esfuerzo le provocó un daño.

Ese mismo carácter nominal explica el hecho de que la construcción de (2) no acepte la voz pasiva ni los tiempos compuestos:

11. *El ser masticado de Juan me molesta.

12. *El haber masticado de Juan me molesta.

También explica que su sujeto solo pueda realizarse por medio de un sintagma preposicional (Bosque, [1990] 2002: 150), como se ve en (13):

\section{El masticar (*Juan/de Juan) me molesta.}

y que solo admita complementos o adjuntos propios del sintagma nominal (Giammatteo y Albano, 2006: 50). De allí, la imposibilidad del circunstancial de modo (continuamente) en (14) y la plausibilidad del adjetivo modificador directo (continuo) en (15), de la aposición (esa manía insistente) en (16) y de la construcción comparativa (como acto reflejo) en (17):

14. *El masticar continuamente de Juan me molesta.

15. El masticar continuo de Juan me molesta.

16. Su masticar, esa manía insistente, es lo único que se oye.

17. El masticar como acto reflejo influye en la sutura de los huesos del cráneo.

Finalmente, y dado que, en muchos casos, los infinitivos se comportan como nominalizaciones, (2) puede ser leído como:

18. La masticación de Juan me molesta.

porque el infinitivo de (2) sirve para nombrar la acción o el evento de masticar.

En síntesis, mientras que la construcción de (1) presenta un caso de INFINITIVO VERBAL, la de (2) está nucleada en torno de un infinitivo que se asemeja ostensiblemente a cualquier

${ }^{2}$ De hecho, esta es otra diferencia notoria entre el comportamiento de la construcción de infinitivo presente en (1) y el de la ejemplificada en (2): mientras que la primera rechaza posesivos y demostrativos, la de (2) los acepta (García Negroni et al., 2004: 260). 
sustantivo. Es por ello que elegimos llamarlo infinitivo sustantivado. En lo que sigue, en primer lugar, estableceremos una clasificación de las distintas construcciones de infinitivo (además de los infinitivos verbales y de los infinitivos sustantivados consideraremos un tercer grupo, el de los FaLSOS INFINITIVOS). Luego, intentaremos dar cuenta del sentido de los diferentes empleos de estos infinitivos en el marco de la semántica argumentativa (Ducrot, 2004; Carel y Ducrot, 2006) y finalmente estableceremos una correlación entre los falsos infinitivos y la aspectualidad semántica.

\section{INFINITIVOS VERBALES, INFINITIVOSSUSTANTIVADOS Y FALSOS INFINITIVOS}

Si bien en general los estudios gramaticales del español se han limitado a distinguir los dos tipos de infinitivos representados por (1) y (2), que recordamos aquí:

1. (El) conocer Juan al ministro le abrió muchas puertas.

2. El masticar de Juan me molesta.

resulta relevante señalar que, además de los infinitivos verbales del tipo de (1) y de los sustantivados del tipo de (2), existe un tercer grupo mucho menos estudiado. Nos referimos a los llamados "infinitivos nominales" (Bosque, 1990) o "falsos infinitivos" (Varela, 1979; Hernanz, 1999). ${ }^{3}$ Este tipo de infinitivos constituye una clase particular en la medida en que puede verse afectada "por los signos morfológicos del número" (Varela, 1979: 539). A modo de ejemplo, considérese (19):

\section{Los haberes de los jubilados han aumentado.}

Al igual que los infinitivos sustantivados, este tipo de infinitivos exige la especificación (cf. la posibilidad de (20) y la imposibilidad de (21)), acepta la cuantificación (cf. 22), la modificación del sintagma adjetivo (cf. 23) y la complementación de un sintagma preposicional (cf. 24):

\section{Los/ Esos/ Mis haberes han aumentado.}

21. *Haberes han aumentado.

22. Ciertos/Algunos/Todos sus haberes han aumentado.

23. Los haberes jubilatorios han aumentado.

24. Los haberes de Juan han aumentado.

${ }^{3}$ Este tipo de infinitivos ha recibido muy escasa atención en las gramáticas y solo se los menciona en notas aisladas. Así, Bello ([1847] 1988: 325), por ejemplo, observa que cuando los infinitivos toman plural "se vuelven sustantivos ordinarios"; Lenz (1920: 186) nota que estos infinitivos son determinados infinitivos que se transforman en sustantivos completos; Gili y Gaya (1948: 165) sugiere que solo los infinitivos que llegan a convertirse en permanentes aceptan el plural y Lamíquiz (1974) señala la existencia de una clase de infinitivos que han llegado a lexicalizarse como sustantivos de lengua, no de discurso (es decir, no por simple interpretación contextual). 
Se observará, sin embargo, que el falso infinitivo se diferencia, aun en singular, de los infinitivos sustantivados. En efecto, mientras que (25) admite como paráfrasis (26), en que el falso infinitivo haber es sustituido por el sustantivo común sueldo, ${ }^{4}$ masticar en (2) solo admite como paráfrasis la nominalización masticación (cf. 27):

\section{El haber de este jubilado ha aumentado.}

26. El sueldo de este jubilado ha aumentado.

\section{La masticación de Juan me molesta.}

$\mathrm{Y}$ es que los falsos infinitivos son verdaderos sustantivos que, como bien afirma Bosque ([1990] 2002: 157), derivan de los verbos correspondientes por medio de un proceso de derivación morfológica.

De todos modos, debe hacerse notar, en primer lugar, que no todos los infinitivos permiten la derivación como falsos infinitivos. De hecho, los infinitivos de (1) y de (2) no la admiten (cf. la imposibilidad de la pluralización en (28) y (29)):

28. *Los conoceres

29. *Los masticares

En segundo lugar, llamamos la atención sobre el hecho de que los falsos infinitivos no constituyen una clase homogénea. En efecto, resulta necesario distinguir dos clases diferentes según su mayor o menor cercanía respecto del sentido de la base verbal:

Falsos infinitivos A

Falsos infinitivos B

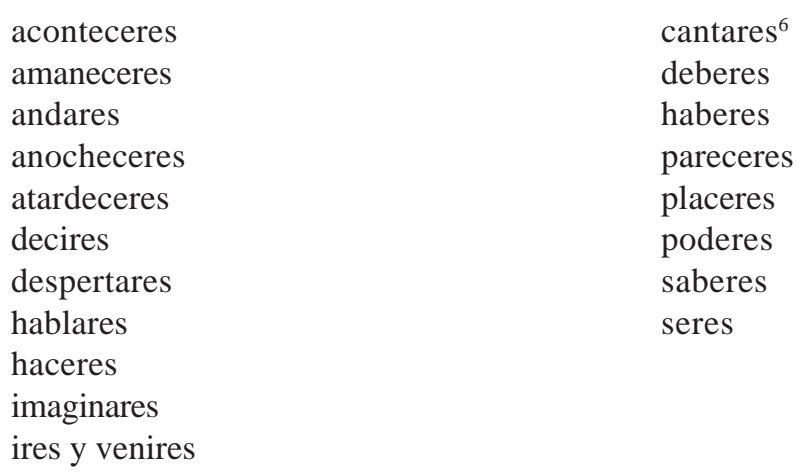

${ }^{4}$ Hacemos notar, sin embargo, que este es fundamentalmente el caso de los que más abajo denominaremos falsos infinitivos de tipo B (haberes, seres, saberes, etc.). En cuanto a los que llamaremos falsos infinitivos de tipo A, algunos admiten la sustitución por un sustantivo común (así, por ejemplo, decires, penares y pesar pueden fácilmente ser parafraseados por sentencias, penas y dolores, respectivamente); otros, en cambio, tienen como paráfrasis una nominalización (así, por ejemplo, aconteceres, padeceres, pensares, sentires, guardan una más estrecha relación con las nominalizaciones respectivas, acontecimientos, padecimientos, pensamientos, sentimientos).

${ }^{6}$ Ver de todos modos infra, nota 7. 
padeceres

penares

pesares

pensares

sentires

vivires

yantares $^{5}$

Cuadro 1. Falsos infinitivos de tipo A y falsos infinitivos de tipo B

Como puede reconocerse, la relación entre los falsos infinitivos A y los verbos de los que ellos derivan resulta más transparente que la de los falsos infinitivos B con los verbos que les sirven de base. Es quizás ello lo que explica por qué Bosque (2002: 157) afirma: "Es difícil definir los sustantivos deber, placer, ser (un ser privilegiado) o parecer (a mi parecer) a partir del significado de los verbos respectivos". Sin dudas, el sentido de amaneceres está más cerca del sentido del verbo amanecer que el de seres del de ser. Pero ello no implica que estos últimos no tengan entre sí un vínculo semántico argumentativo que explique la derivación. Volveremos sobre esta cuestión más adelante (cf. § 3).

En suma, y a la luz de lo hasta aquí señalado, puede sostenerse que:

* los infinitivos verbales, los infinitivos sustantivados y los falsos infinitivos, ejemplificados respectivamente en (1), (2) y (19), representan tres tipos diferenciados de construcciones de infinitivo con valor nominal en una escala progresiva de sustantivación;

* la mayoría de los infinitivos admite ocurrir en las construcciones del tipo (1) y (2), como se ve, por ejemplo, en las ocurrencias de beber en (30) y (31). Obsérvese que mientras que en (30), (el) beber funciona como infinitivo verbal (cf. la opcionalidad del determinante $e l$ y la presencia del circunstancial de modo hasta la embriaguez), en (31) su beber lo hace como un infinitivo sustantivado (cf. la obligatoriedad del determinante $s u$ y la presencia del adjetivo incontrolado como modificador directo):

30. Para Silvio Sánchez, que cursa el grado doce en la Escuela Braddock, de Enseñanza Secundaria y piensa estudiar ciencias gráficas en el Instituto Tecnológico de Rochester, New York, "el beber hasta la embriaguez es incorrecto, en cuanto puede causar la muerte no sólo de quien conduce el vehículo, sino también de quien le acompaña". (CREA: Diario de las Américas, 16/04/1997: Certamen de afiches contra el alcoholismo entre los jóvenes)

\section{Aunque en apariencia sea un acto inhumano dejar sola a una persona querida cuando} está atravesando una circunstancia difícil de su vida, en ocasiones se le ayuda mucho

${ }^{5}$ La existencia de todos estos falsos infinitivos ha sido constatada en Real Academia Española: Banco de datos (CREA) [en línea]. Corpus de referencia del español actual. http://www.rae.es. Al mismo corpus pertenece, asimismo, la casi totalidad de los ejemplos reales que ilustran el presente trabajo y así consta en cada uno de ellos. 
porque se le enfrenta a la consecuencia que ha traído su beber incontrolado. (CREA: Barriguete Castellón, A., Lo que el vino se llevó, México, Diana, 1996)

* solo algunos infinitivos admiten la derivación como falsos infinitivos: beber, por ejemplo, no la admite (como permite inferirlo la inexistencia de casos de beberes en el Corpus de referencia del español actual). Los falsos infinitivos constituyen formas nominales puras que funcionan de manera autónoma en relación con los verbos que les han servido de base; se trata de formas que han adquirido un sentido propio y bien determinado. Prueba de ello es que admiten la modificación morfológica representada por el plural, impensable para los infinitivos, que, como se sabe, son invariables.

En el próximo apartado, y luego de pasar revista brevemente a ciertos conceptos clave de la teoría semántica argumentativa, intentaremos, dentro de ese marco, dar cuenta de las diferencias entre los infinitivos verbales, los infinitivos sustantivados y los falsos infinitivos.

\section{ARGUMENTACIÓN,SENTIDOE INFINITIVOS}

Según la Teoría de la argumentación en la lengua (TAL), teoría semántica no referencialista, el sentido de las entidades lingüísticas (enunciados, palabras) no está constituido por las cosas, los hechos o las propiedades que ellas denotan, ni por los pensamientos o creencias que las suscitan o que ellas sugieren, sino por ciertos discursos que les están asociados. El sentido es caracterizado así en términos de argumentación, esto es, en términos de la inserción de los enunciados en los encadenamientos discursivos.

Ahora bien, si en su versión estándar conocida como Teoría de los Topoi (cf. Ducrot, 1987; Anscombre, 1995), la TAL se limitaba exclusivamente a los encadenamientos resultativos en por lo tanto, su desarrollo más reciente, la Teoría de los bloques semánticos (TBS), ha puesto en evidencia la necesidad de ampliar la noción de argumentación y de considerar como básicos no solo los encadenamientos normativos, es decir aquellos que pueden manifestarse a través de conectores del tipo de por lo tanto, si... entonces, es por ello (realizaciones de un conector abstracto POR LO TANTO, abreviado como PLT), sino también un segundo tipo de discursos llamados transgresivos y señalados por conectores del tipo de sin embargo, aunque, aun cuando, a pesar de que (realizaciones de un conector abstracto SIN EMBARGO, abreviado como SE).

La razón por la que se les otorga actualmente a estos dos tipos de encadenamientos ese rol privilegiado es que en ellos "los enunciados encadenados no tienen una realidad semántica que podría comprenderse haciendo abstracción de su encadenamiento" (Ducrot 2004: 365). Estrictamente discursivos, estos encadenamientos son irreductibles a relaciones entre propiedades independientes. Así, por ejemplo, en:

\section{María tiene hijos: es feliz.}

el sentido del primer segmento es solo determinable por el hecho de que trae aparejada la felicidad expresada después del por lo tanto en el segundo y no, por ejemplo, el desvelo o 
las preocupaciones que los hijos provocan en nosotros. Por su parte, la felicidad evocada en el segundo miembro es aquella que se presenta como una especie de consecuencia natural de tener hijos y no, pongamos por caso, la que podría proporcionar el haber ganado la lotería o la posesión de dinero y de bienes, como ocurre en (33):

33. María es rica: es feliz.

Considérense ahora (34) y (35).

34. María tiene hijos y sin embargo no es feliz.

35. Aunque es rica, María no es feliz.

Como puede constatarse, al igual que en (32), la maternidad de la que se trata en (34) es la que lleva a la felicidad y la felicidad evocada es la debida a la maternidad. En (35), por su parte, y al igual que en (33), la riqueza de la que se habla es aquella que puede llevar a la felicidad (y no, por ejemplo, a cuidarse de posibles secuestros exprés) y la felicidad, solo la relacionada con la que puede dar el dinero. La TBS afirma así que los encadenamientos (32) y (34), por un lado, y (33) y (35) por el otro, pertenecen a los mismos bloques semánticos (el de la felicidad-de-la-maternidad, en el caso de (32) y (34), y el de la felicidad-de-lariqueza en el caso de (33) y (35)). Pero mientras que (32) y (33) aplican esos bloques bajo su aspecto normativo (i.e., dos segmentos unidos mediante el conector abstracto PLT):

\section{maternidad PLT felicidad}

dinero PLT felicidad

(34) y (35) lo hacen bajo su aspecto transgresivo (i.e., dos segmentos unidos mediante el conector abstracto SE seguido de una negación):

maternidad SE Neg-felicidad

dinero SE Neg-felicidad

Ahora bien, si según la tesis central de la TBS, el sentido de una entidad lingüística, palabra o enunciado, reside en las argumentaciones normativas en PLT y transgresivas en SE que esa entidad evoca, dos son los modos según los cuales dicha entidad puede evocar los encadenamientos argumentativos que constituyen su sentido. Estos dos modos reciben el nombre de argumentación externa (AE) y argumentación interna (AI).

Según sostienen Carel y Ducrot (2006), la AE de una determinada entidad lingüística está constituida por los discursos argumentativos, normativos y transgresivos, en los que esa entidad interviene en tanto primero o segundo segmento. Se dice así, por ejemplo, que forman parte, entre otros, de la $\mathrm{AE}$ del adjetivo feliz los discursos:

36. María es feliz, por lo tanto siempre sonríe. 37. María es feliz, sin embargo nunca sonríe.
AE a la derecha

$\mathrm{AE}$ a la derecha 
38. María tiene hijos: es feliz.

39. María no tiene hijos, sin embargo es feliz.
AE a la izquierda ${ }^{7}$

AE a la izquierda

Pero la AE no resulta suficiente para definir el sentido de las entidades lingüísticas. Y es por ello que la teoría introduce la noción de argumentación interna (AI). Se trata en este caso de los discursos argumentativos, normativos o transgresivos, en los que la entidad lingüística no interviene y que constituyen una especie de paráfrasis de la entidad. Así, por ejemplo, a la luz de la definición propuesta por el DRAE, podría afirmarse que la AI de felicidad es:

\section{posesión de un bien PLT satisfacción}

Decir felicidad evoca, en efecto, una relación normativa en por lo tanto entre la indicación de la posesión de una cosa o bien (en los ejemplos (32) a (35), los hijos o el dinero) y la satisfacción que dicha posesión provoca o trae aparejada.

Existe una diferencia suplementaria entre las AE y las AI. Las primeras se caracterizan por el hecho de que si una entidad $e$ contiene como parte de su AE el discurso $e$ conector $Y$, también contendrá en ella el discurso converso e conector' Neg. $Y$. (cf. por ejemplo, los discursos conversos (36)-(37) y (38)-(39) que constituyen la AE de feliz). No ocurre lo mismo con las AI. En efecto, si en la AI de una entidad $e$ se encuentra un discurso $X$ conector $Y$, no se encontrará en esa misma AI el discurso converso $X$ conector' $N e g-Y$. Este segundo discurso permitirá definir la AI de la negación de $e$. Así, una buena descripción de la AI de infelicidad podría ser:

\section{posesión de un bien SE Neg-satisfacción}

En suma, y tal como señalan Carel y Ducrot (2006), para definir la significación de una determinada entidad lingüística, debe asociársele una familia de discursos argumentativos, normativos o transgresivos. Luego, se clasifican esos discursos en dos sub-familias, según que esa entidad lingüística intervenga o no y, si interviene, se considera si lo hace en tanto primero o segundo miembro de ese discurso argumentativo. Aquellos discursos en los que la entidad lingüística interviene constituyen la argumentación externa de la entidad; los otros, su argumentación interna.

Munidos de estas herramientas teóricas, propondremos a continuación una breve descripción semántico-argumentativa de los distintos tipos de infinitivos que nos conciernen.

Comencemos por las construcciones del tipo de (1) y (2). Si bien desde el punto de vista de la función que cumplen en la oración, estos infinitivos revisten, como vimos, una

${ }^{7}$ A diferencia de la Teoría de los Topoi, que solo tomaba en consideración las argumentaciones externas y, entre ellas, solo las $\mathrm{AE}$ a la derecha, es decir aquellas en las que la entidad interviene como primer segmento de la argumentación, la TBS también toma en consideración las AE a la izquierda, es decir aquellas en las que la entidad interviene como segundo segmento. Para un recorrido histórico de los fundamentos teórico-metodológicos de la semántica argumentativa, podrá consultarse García Negroni, M. (2008). 
condición nominal, lo cierto es que mantienen su funcionamiento verbal a pesar de haber quedado borradas en ellos las huellas de la flexión. En este sentido, afirmamos que los infinitivos verbales y los infinitivos sustantivados conservan las argumentaciones internas y desarrollan, según el contexto en el que aparecen, (cualquiera de) las argumentaciones externas, tanto a la derecha como a la izquierda, de los verbos de los que proceden. En el caso del infinitivo sustantivado, la selección de las AE aparece fundamentalmente determinada por el modificador o complemento nominal que lo acompaña como tal.

Tomemos como ejemplo el caso del verbo vivir (para distinguirlo de las construcciones de infinitivo, aludiremos a él mediante una de sus formas conjugadas: vive). Como puede constatarse en (40) y (41):

40. La planta vive varios años y fructifica anualmente.(CREA: Fuentes Yagüe, J.L. Iniciación a la botánica, Madrid, Ediciones Mundi-Prensa, 2001)

41. Hemos vivido momentos de inquietud. (Diccionario de la RAE)

la AI de vive puede ser definida como:

AI de vive: $\quad$ existe PLT puede sentir

Llamamos aquí la atención sobre el hecho de que, tal como prevé la TBS, el discurso converso:

\section{existe SE Neg-puede sentir}

no forma parte de la AI de vive sino de su negación: no vive, en el sentido de no tiene vida (cf., por ejemplo, las plantas viven/tienen vida pero los minerales, no).

En cuanto a las AE de vive, pueden incluirse en su sentido, entre otras, las siguientes:

AE de vive en (40): $\quad$ vive PLT se reproduce

vive PLT deja huellas

Neg-murió PLT vive

Neg-desplazamiento SE vive

AE de vive en (41): $\quad$ vive PLT adquiere experiencia

vive PLT deja huellas

Neg-murió PLT vive

Consideremos ahora el caso del infinitivo verbal (el) vivir, presente en (42), y del infinitivo sustantivado el vivir cotidiano, que aparece en (43):

42. La señora Cruz Flores manifestó que el problema se agudiza cada vez más, aunque para ella no es una preocupación, pues el vivir por tantos años contiguo al crematorio la ha hecho acostumbrarse a los malos olores. (CREA: El Salvador Hoy, 04/07/1997) 
43. Y no es utopía porque así sucede en los países de nuestro entorno a los que por voluntad propia hemos unido nuestro destino, con los que compartimos ideas, pensamientos, sentimientos, con los que hemos constituido alianza tanto para el vivir cotidiano, en lo económico y en lo político, en la creación artística y científica, como en la disposición y preparación para la común defensa. (CREA: ABC, 28/05/ 1989)

En ambos casos, y tal como puede constatarse, (el) vivir y el vivir cotidiano conservan la AI del verbo conjugado, a saber:
AI de (el) vivir:
$\underline{\text { existe PLT puede sentir }}$

En cuanto a las AE, y dado el contexto de (42), el infinitivo verbal (el) vivir desarrolla las siguientes:

AE de (el) vivir: $\quad$ vive PLT adquiere experiencia

Neg-murió PLT vive

Neg-desplazamiento SE vive

En tanto, el vivir de (43), y dada la determinación establecida por sus especificadores (el y cotidiano), desarrolla:

AE de el vivir cotidiano: $\quad$ vive PLT deja huellas

$\underline{\text { Neg-murió PLT vive }}$

Diferente es el caso de los falsos infinitivos. En efecto, dado su mayor grado de sustantivación, sostenemos que estos nombres no conservan, como interna, la AI del verbo del que derivan, sino que retienen una de sus AE para lexicalizarla y transformarla en su propia AI. Y lo hacen de manera diferenciada según se trate de falsos infinitivos de tipo A o de tipo B. Así, mientras que los de tipo A seleccionan una de las AE a la derecha del verbo base como su AI, los de tipo B lo hacen con una de las AE a la izquierda del verbo.

Consideremos a modo de ejemplo (44):

44. Tik, la palabra que brilla en el centro de los decires y los vivires de estas comunidades mayas, significa nosotros. (CREA: Galeano, E., Bocas del tiempo, Madrid, Siglo XXI, 2004)

donde el sentido del falso infinitivo de tipo A los vivires puede ser descripto mediante la siguiente AI:

AI de los vivires: $\quad$ vive PLT deja huellas

que es, como recordamos, una de las $\mathrm{AE}$ a la derecha de vive. Dicho en otros términos, los vivires remite siempre a los modos de vivir (en (44), a los de las comunidades mayas), y, en 
este sentido, a las huellas que esos modos dejan y que pueden ser reconocidas por la historia y la posteridad.

De igual manera -sostenemos-, los otros falsos infinitivos de tipo A seleccionan una de las AE a la derecha del verbo base para constituir su AI. A modo de ejemplo, y como puede verificarse en los siguientes ejemplos del corpus (cf. (45)-(48)):

45. Ya en sus quehaceres, y de acuerdo con los decires de la propia víctima en la comisaría, María fue "requerida de amores" por un enamoradizo Blas, quien ahí nomás le hizo “formal promesa de matrimonio". (CREA: La Nueva Provincia, 15/10/1997).

46. Muera el amor, decía, abajo la tiranía de los sentires. (CREA: Merino, J. Novela de Andrés Choz, Madrid, Mondadori, 1987)

47. Se pretende, con esta medida, terminar con las larguísimas colas y con los padeceres de quienes deben esperar por sus haberes soportando los rigores del clima. (CREA: La Nueva Provincia, 06/05/1997)

48. Ha sido como el triunfo del PRD en Iguala después de las derrotas de Michoacán y Nayarit: alivio a los pesares y árnica sobre los moretones. (CREA: Proceso, 27/10/1996)

las AI de estos falsos infinitivos corresponden a una de las AE a la derecha de los verbos de los que ellos derivan:

AI de los decires: $\quad$ dice PLT se hace oír (AE a la derecha de dice)

AI de los sentires: $\quad$ siente PLT demuestra emoción (AE a la derecha de siente)

AI de los padeceres: padece SE soporta (AE a la derecha de padece)

AI de los pesares: $\quad$ pesa SE lleva (AE a la derecha de pesa)

En cuanto a los falsos infinitivos de tipo B y para poder dar cuenta de ellos, tomemos ahora el caso del verbo saber (nuevamente, para distinguirlo de las construcciones de infinitivo, aludiremos a él mediante una de sus formas conjugadas: sabe). ${ }^{8}$ Como puede constatarse en (49) y (50):

${ }^{8}$ Si bien en el caso de vive no resulta posible la derivación de un falso infinitivo de tipo $\mathrm{B}$, no nos sentimos en condiciones de afirmar que no existan falsos infinitivos de ambos tipos derivados de una misma base. De hecho, aunque resultan ambiguos, es muy probable que algunos de los ejemplos del corpus correspondientes al falso infinitivo cantares (que en nuestro listado aparece incluido dentro de los infinitivos de tipo B) pertenezcan al grupo de los falsos infinitivos de tipo A. Así, por ejemplo, en: "A la puesta del sol cesaba el areito y se iban todos para sus casas, y lo mismo hacían en cada casa, cada uno delante de sus dioses; había un gran ruido en todo el pueblo por razón de los cantares y del tañer en cada casa." (CREA: Aguilera, C. El arte oficial tenochca. Su significación social, México, UNAM, 1985), la lectura de cantares (que podría ser descripta mediante la AI: canta PLT se escucha) no parece ser la misma que en los cantares de gesta que, en tanto falso infinitivo de tipo B, tiene como AI: es una historia antigua PLT se canta. 
49. ¡Yo maté a Rebecca! Yo y Raquel lo hicimos, inventamos una historia pero ella no pudo con su conciencia. Sí, la maté. ¿Y ahora? ¿Qué hacemos ahora que ya sabe la verdad? (Burlón) ¿Inventamos otra mentirita? (CREA: Ramos-Perea, R. Obsesión. Galante (Mayagüez), Ediciones Gallo, 1989)

50. Majestad, como dijo San Agustín, el rey sabe de todo, pero sobre todo, sabe latín, y si más no sabe es que más no cabe. (CREA: Márquez, J. Mientras que Némesis duerme, Madrid, Fundamentos, 1990)

sabe puede ser descripto mediante las siguientes argumentaciones:

AI de sabe: $\quad \quad$ posible de aprender in toto PLT se aprendió

AE de sabe en (49): $\quad$ sabe PLT puede responder

fue informado PLT sabe

$\underline{\text { sabe PLT se da cuenta }}$

susceptible de ser conocido PLT sabe

AE de sabe en (50): $\quad$ sabe PLT puede responder $\underline{\text { conocimiento susceptible de ser profundizado PLT sabe }}$

Por su parte, y tal como puede observarse en (51) y (52), tanto el infinitivo verbal (el) saber como el infinitivo sustantivado el saber de lo inevitable conservan la misma AI que sabe y desarrollan, según el contexto particular en el que cada uno aparece, (algunas de) sus AE (a la derecha y a la izquierda):

51. No es para nadie algo ajeno el saber los problemas internacionales que tenemos para mantener el crecimiento económico. Tampoco la grave crisis que sufrimos a inicios del sexenio. (CREA: Oral. Sesión pública ordinaria de la Honorable Cámara de Senadores, celebrada el miércoles 9 de septiembre. México)

52. El conocimiento no es sólo el saber de lo inevitable, sino también el dolor, la resignación, el sufrimiento. (CREA: Vásquez, E., Libertad y enajenación, Caracas, Monte Ávila Editores, 1987)

AI de (el) saber/el saber lo inevitable: posible de aprender in toto PLT se aprendió

$\mathrm{AE}$ de (el) saber en (51): sabe PLT puede responder sabe PLT se da cuenta fue informado PLT sabe

AE de el saber de lo inevitable en (52): $\quad$ sabe PLT puede responder $\underline{\text { sabe PLT se da cuenta }}$ susceptible de ser conocido PLT sabe 
Finalmente, y en lo que concierne al falso infinitivo de saber, los saberes, que, como queda dicho, pertenece al grupo de los falsos infinitivos de tipo B, (cf. (53)):

53. Theodor Lipps, a principios de siglo y en el ámbito de los saberes estéticos, dejó escrito que los dos componentes fundamentales de la empatía son la "proyección" y la "imitación”. (CREA: ABC Electrónico, 01/10/1997)

se constatará que su AI corresponde a la lexicalización de una de las externas a la izquierda de sabe:

AI de los saberes: $\quad$ conocimiento susceptible de ser profundizado PLT sabe

Y otro tanto ocurre -sostenemos- con los otros falsos infinitivos de tipo B: en todos los casos, sus AI están constituidas por la selección de una de las AE a la izquierda del verbo de los que derivan. Así, por ejemplo, y tal como lo muestran los siguientes ejemplos tomados del corpus:

54. Nicolás Mandaza, fallecido en noviembre de 1994, figura en la planilla de liquidación de haberes de la Legislatura Provincial de Formosa. (CREA: El Tiempo, 11/11/1996)

55. Por citar un bello ejemplo de aquellos cantares, cuya música se ha perdido en el tiempo, citaré para concluir un canto que se asocia con una danza previa al sacrificio de los prisioneros humanos, mediante mortales flechas, lo que da una idea de la riqueza literaria del Chilam Balam. (CREA: La información : "Sacerdote Jaguar", 1996)

56. ¡No son un peluche, tienen más vida que muchos seres humanos! (CREA: El País, 04/ 10/2001)

57. Y aquí en la ley sí se habla de algunos deberes, pero que nunca estos deberes van a ser suficientes para menoscabar la protección de los derechos de ellos. (CREA: Oral, Fox en vivo, Fox contigo, 04/11/00, Radio ACIR)

58. Educado para la guerrilla, el joven burgués venezolano descubre la guerra. Un tránsito hacia la carrera que le convierte verdaderamente, la de terrorista. Una manera de continuar la guerra sin romper con los placeres urbanos dignos de su origen. Porque a Illich le va la buena vida. (CREA: La Vanguardia, 18/08/1994)

las AI de esos falsos infinitivos (de tipo B) pueden describirse como:

AI de haberes: $\quad$ se trabajó PLT hay paga (AE a la izquierda de hay)

AI de cantares: $\quad$ es una historia antigua PLT se canta (AE a la izquierda de canta)

AI de seres:

AI de deberes: existe PLT es (AE a la izquierda de $e s$ )

AI de placeres: $\quad$ es disfrutable PLT le place (AE a la izquierda de place) 
Ahora bien, afirmamos más arriba que la relación entre los falsos infinitivos y los verbos de los que ellos derivan resulta menos transparente en el caso de los falsos infinitivos de tipo B. La caracterización de la AI de los falsos infinitivos de tipo A en términos de lexicalización de una de las AE a la derecha del verbo base y la de los falsos infinitivos de tipo B en términos de lexicalización de una de las AE la izquierda del verbo base permite dar cuenta, creemos, de la mencionada afirmación.

En efecto, en la medida en que la $\mathrm{AE}$ a la derecha toma como punto de partida el verbo base, la relación entre este y el segundo miembro del encadenamiento parece natural y fluida. La lexicalización, en cambio, de la $\mathrm{AE}$ a la izquierda requiere recuperar como primer segmento un elemento que no es la base verbal sino algo presupuesto por ella. Es, por ejemplo, lo que ocurre en el caso de saberes (cf. (53)), donde el nexo entre sabe y los saberes exige presuponer la posibilidad de la profundización de un cierto conocimiento. No se trata pues de que estos sustantivos no posean relación productiva con el verbo cuya forma comparten ni que sea difícil definirlos a partir del significado de los verbos respectivos (Bosque, 2002: 157) sino, en todo caso, de reconocer la existencia de la AE a la izquierda para poder determinar el vínculo entre ambos.

\section{FALSOS INFINITIVOSYASPECTUALIDAD SEMÁNTICA}

Hay un dato más, todavía, que nos llama la atención al analizar los falsos infinitivos y es su relación con la aspectualidad semántica.

Como decíamos más arriba, mientras todos los verbos admiten ocurrir en construcciones como las de (1) y (2), es decir, como infinitivos verbales o infinitivos sustantivados, solo algunos pocos derivan falsos infinitivos, tal cual lo pone de manifiesto el breve -si bien, creemos, bastante completo- listado que hemos registrado aquí (cf. cuadro 1, en §2).

Un rasgo aspectual constante recurre en esa lista de falsos infinitivos: se trata de la homogeneidad/propiedad. En efecto, varios de estos infinitivos son estativos del tipo de los puros (Ramírez Gelbes, 2008) ${ }^{9}$; los restantes, son actividades (Vendler, 1967). En ambos casos, se trata de situaciones caracterizadas como homogéneas y, básicamente, atélicas. Así, frente a los seres o los saberes (falsos infinitivos derivados de estativos puros) y a los cantares o los ires y venires (falsos infinitivos derivados de actividades), no encontramos * los estares ni *los conoceres (que serían falsos infinitivos derivados de estativos delimitados), ni tampoco *los hundires ni *los estallares (que serían falsos infinitivos derivados de realizaciones o logros, i.e., télicos), por ejemplo.

Esto que afirmamos no significa, claro está, que todos los estativos puros y todas las actividades hayan de derivar falsos infinitivos. Como sostiene Bosque (2002: 113) en relación con la sustantivación a partir de adjetivos, debe reconocerse que la sustantivación léxica de un infinitivo puede ser excluida por la existencia en la lengua de un sustantivo que cumpla ya ese papel o, en otras palabras, que no todas las derivaciones se dan de manera completa. Pero parece bastante notorio que solo los verbos homogéneos admitan esta

\footnotetext{
${ }^{9}$ Llamamos puros a los estativos del tipo de ser o de saber, que no parecen contener ninguna noción de límite en su descripción. Los distinguimos de los delimitados, del tipo de estar y conocer, que sí contienen esa noción.
} 
derivación mientras que los que focalizan la delimitación en su sentido la rechacen. Y la razón para que esto sea así es el hecho de que los homogéneos tienden a sustantivar su homogeneidad, representada por la forma no personal del infinitivo, poniendo el énfasis en la situación completa evocada por el verbo y transformándola en una propiedad.

Una única excepción al respecto parece ser el caso de despertares. Por cierto, se trata de un falso infinitivo que deriva, de acuerdo con toda la bibliografía aspectual, de un verbo télico o delimitado: despertar (aunque, sobre todo, despertarse) conlleva la idea de un límite que debe ser alcanzado para que el evento denotado por el infinitivo se concrete. Dicho en otras palabras, si algún suceso interrumpiese el evento (por ejemplo, una muerte súbita) no se llevaría a cabo el despertar(se). Se observará no obstante que en tanto falso infinitivo, y tal como se constata en los distintos ejemplos del corpus, despertares no pone el foco en el límite puntual de la situación en que se produce el resultado de estar despierto. Antes bien, despertares focaliza ya en el desarrollo del evento evocado por el verbo (cf. 59 y 60), ya en la iteración del proceso (cf. 61 y 62), favoreciendo así una lectura homogénea de propiedad:

59. Lo veo todo claro y ordenado, mientras giro en la cama, alargo los brazos y me desperezo para levantarme. Sin cansancio, sin sensación negativa alguna, con ánimos de actuar en todo lo que acabo de plantearme, ordenadamente, en medio de un agradable relajo. ¿Qué afortunado soy! ¡Ojalá mis despertares sean siempre así! Sin malhumores. Disfruto de la sensación de estar de nuevo vivo después de unas adormecidas horas con la impresión de no existir. (CREA: Llongueras, L., Llongueras tal cual. Anécdotas y recuerdos de una vida. Barcelona, Planeta, 2001)

60. Además de recibir las confesiones y sectas europeas, en Estados Unidos aparecen nuevas versiones religiosas, y, respondiendo a un entusiasmo milenarista, se producen “despertares” periódicos, cada veinticinco o treinta años, que dan lugar a nuevas iglesias. (CREA: Zaragoza, G., Las grandes religiones (II), Madrid, Anaya, 1993).

61. Encontramos una paciente de aspecto demacrado, con ojeras, piel seca, cabello se mostraba seco y con tendencia a caída abundante, extremidades con tono azulado al igual que labios, manos frías al tacto, gran dificultad para mantenerse relajada, así como para mantener la postura. La espalda de la paciente estaba cubierta de vello.

Se mostraba triste, con sentimientos de un intenso miedo al rechazo o abandono, sus palabras denotaban baja autoestima, altos niveles de ansiedad, irritable, el sueño había últimamente empeorado y presentaba despertares frecuentes. (CREA: Revista de Psiquiatría y Psicología del Niño y del Adolescente, $\mathrm{n}^{\circ}$ 2, 2002)

62. Esta alteración afecta por igual a ejecutivos que a amas de casa, quienes se quejan de tener grandes dificultades para quedarse dormidos y, a veces, frecuentes despertares nocturnos. El problema suele tener su origen en un trastorno médico o en un episodio de estrés agudo -conflictos laborales, conyugales, de autoestima...- que, a pesar de haber sido superado, ha dejado como secuela un grave trastorno de sueño. (CREA: Estivill, E.; de Béjar, S., ¡Necesito dormir! El insomnio sí tiene solución, Barcelona, Plaza y Janés, 1997) 
En suma, creemos que la hipótesis de la existencia de una relación estrecha entre falsos infinitivos y homogeneidad/propiedad puede ser mantenida para dar cuenta de por qué solo cierto tipo de verbos permite la derivación de falsos infinitivos en español.

\section{CONCLUSIONES}

En este trabajo, nos hemos ocupado de analizar distintas construcciones nominales de infinitivo en español. Partimos para ello de la consideración de las construcciones más frecuentemente consideradas en la bibliografía especializada: la del infinitivo verbal (que, al igual que los predicados verbales, admite sujeto en nominativo, objeto directo en acusativo y otros adjuntos) y la del infinitivo sustantivado (que, en tanto construcción sustantiva, solo acepta modificadores nominales).

En segundo lugar, observamos la existencia de una tercera construcción de infinitivos nominales, que hemos denominado, siguiendo a Varela y a Hernanz, falsos infinitivos. Estos infinitivos aceptan la pluralización al modo de los sustantivos ordinarios (cf. los decires, los ires y venires, los haberes, los seres, etc.) y, como ellos, entran en todas las construcciones nominales. Pero no solo esto. Tal cual intentamos poner de manifiesto en este trabajo, dos son los tipos de falsos infinitivos que pueden ser reconocidos: los falsos infinitivos de tipo A y los falsos infinitivos de tipo B. Mientras que los primeros se acercan más al sentido de las nominalizaciones de los verbos de los que derivan, los segundos parecen más alejados de ellos. Sin embargo, esto es así solamente si no se considera su descripción argumentativa.

En efecto, y a la luz de los principios de la teoría de los bloques semánticos (en particular los relacionados con las argumentaciones externas e internas), fue posible constatar que, mientras el sentido de los falsos infinitivos de tipo A se describe a partir de las AE a la derecha del verbo base, la AI de los falsos infinitivos de tipo B se define a partir de las AE a la izquierda de los verbos correspondientes. Pudimos dar cuenta así de la aparente mayor distancia semántica que existe entre este último tipo de infinitivos y los verbos de los que ellos derivan. Y para poder brindar una explicación de la escasa productividad de los falsos infinitivos, propusimos relacionarlos con la aspectualidad: solo los verbos que contienen el rasgo homogeneidad/propiedad (como los estativos puros o las actividades) pueden desarrollar falsos infinitivos.

En definitiva, las construcciones de infinitivo representan un espacio interesante de investigación que demuestra por un lado la capacidad explicativa de la TBS en tanto teoría semántica léxica y que por el otro permite arrojar luz sobre los alcances de las nociones relativas a la aspectualidad semántica.

\section{BIBLIOGRAFIA}

ANSCOMBRE, J.-C. (org.) (1995). Théorie des Topoi. París: Kimé.

BELLO (1847). Gramática de la lengua castellana destinada al uso de los americanos. Madrid: Arco Libros, 1988. 
BOSQUE, I. (1990). Las categorías gramaticales. Madrid: Cátedra, 2002.

CAREL, M. y DUCROT. O. (2006). La semántica argumentativa. Una introducción a la teoría de los bloques semánticos. Buenos Aires: Colihue.

DUCROT, O. (1987). "Argumentation et topoï argumentatifs", Actes de la 8ème rencontre des professeurs de français de l'enseignement supérieur, Helsinski, p. 27-57, 1988. Traducido al español en Lenguaje en contexto, vol.1, $\mathrm{n}^{\circ} 1-2$, p. 63-84.

. (2000). "La elección de las descripciones en semántica argumentativa léxica", Revista iberoamericana de Discurso y Sociedad, vol. 2, n 4, p. 23-45.

. (2004). "Sentido y argumentación". En Arnoux, E. y M.M. García Negroni (comp.) Homenaje a Oswald Ducrot (pp. 359-370). Buenos Aires: Eudeba.

GARCÍA NEGRONI, M.M. et al. (2004). El arte de escribir bien en español. Buenos Aires: Santiago Arcos editor.

GARCÍA NEGRONI, M. M. (2008). “Argumentación y descripción semántica. Acerca del sentido en la semántica argumentativa". Actas de la Conferencia internacional "Lógica, argumentación y pensamiento crítico", Santiago: Chile, 8 al 11 de enero.

GiAmMATEO, M y AlBANO, H. (2006). Cómo se clasifican las palabras. Buenos Aires: Littera.

GILI Y GAYA, S. (1948). Curso superior de sintaxis española. Barcelona: Spes.

HERNANZ, M. L. (1999). "El infinitivo”. En Bosque, I. \& V. Demonte (eds.) Gramática descriptiva de la lengua española (pp. 2197-2356). Madrid: Espasa.

LAMÍQUIZ, V. (1974). Lingüística española. Sevilla: PUS.

LENZ, R. (1920). La oración y sus partes. Madrid: Centro de Estudios Históricos.

RAMÍREZ GELBES, S. (2008). "La Teoría de los Bloques Semánticos y la aspectualidad: los 'externalizados'”, Analecta Malacitana, 25, p.81-105. [disponible en: http://www.anmal.uma.es/ numero25/Indice.htm]

VARELA, S. (1979). "Los falsos infinitivos", BRAE : LIX, p. 529-551.

VENDLER, Z. (1967). "Verbs and Times". Philosophical Review, 56, p. 143-160. 